\title{
Study of Parallel Linear Solvers for Three-Dimensional Subsurface Flow Problems
}

\author{
Hung V. Nguyen, Jing-Ru C. Cheng, and Robert S. Maier \\ U.S. Army Engineer Research and Development Center \\ Major Shared Resource Center \\ 3909 Halls Ferry Road \\ Vicksburg, MS 39180-6199 \\ \{Hung.V.Nguyen, Ruth.C.Cheng, Robert.S.Maier\} @usace.army.mil
}

\begin{abstract}
The performance of an iterative method for solving a system of linear equations depends on the structure of the system to be solved and on the choice of iterative solvers in combination with preconditioners. In this paper, the performance of a specified set of linear solvers and preconditioners provided by PETSc and Hypre is evaluated based on three data sets from subsurface finite element flow models. The results show that simple preconditioners are robust but do not enable convergence behavior that scales with problem size. They also show that it is important to choose an appropriate type of solver for different kind of simulations.
\end{abstract}

Keywords: Sparse parallel solvers, iterative solvers, PETSc, Hypre, and finite element.

\section{Introduction}

Simulations of groundwater [3] and watershed [1] flow require the solution of a nonlinear system of partial differential equations (PDE). Discretization of the PDE spatial domain on a finite-element mesh results in a set of time-dependent, nonlinear algebraic equations that may be solved by a Newton or Modified Picard algorithm. These algorithms require the solution of one or more large sparse systems of linear equations at each time-step, of the form,

$$
\mathbf{A x}=\mathbf{b}
$$

where $\mathbf{A}=\left[a_{i j}\right]$ is an $n \times n$ matrix and $\mathbf{b}$ a given right-hand-side vector.

WASH123 is a simulation code used for modeling time-dependent surface and subsurface flows, and the coupling between the two flow regimes [2]. WASH123 uses a modified Picard algorithm to solve the nonlinear problem of three-dimensional (3-D) subsurface flow, and the resulting linear systems can be shown to be symmetric and positive definite (SPD) in all cases. Also, the matrix A may vary over the course of a time-dependent simulation depending on the forcing, coupling, and boundary conditions. A significant part of the computation time of a WASH123 simulation is spent solving the linear system [5], [6]. Therefore, the performance of linear solvers is of great interest. 
Iterative methods are most often used for linear problems involving a large number of variables because of the memory and scalability restrictions of direct methods, such as LU decomposition. Krylov subspace methods are an important class of iterative solution solvers [4], [8]. This class includes the Conjugate Gradient (CG) method, which is robust for SPD matrices. Tracy and Gavali have previously tested a wide range of iterative solvers [4] on matrices arising from the FEMWATER code, a predecessor of WASH123. Their results suggest that CG remains a competitive method for matrices in the range of a few million unknowns, but leaves open the question of the most effective preconditioner. In practice, combining a Krylov subspace method with a preconditioner is essential, especially for an ill-conditioned linear system. Therefore, this study tests the effectiveness of iterative solvers combined with various preconditioners to determine those most effective for subsurface and coupled watershed flow applications.

The convergence criterion for an iterative method is typically tied to the residual, $\mathrm{r}^{\mathrm{k}}=\mathbf{b}-\mathbf{A} \mathbf{x}^{\mathrm{k}}$, where superscript $\mathrm{k}$ denotes the $\mathrm{k}^{\mathrm{th}}$ iteration. The PETSc convergence criterion was used:

$$
\left\|\mathbf{r}^{\mathrm{k}}\right\|_{2}<\varepsilon\|\mathbf{b}\|_{2}
$$

where $\varepsilon$ is relative tolerance, $\varepsilon_{=} 10^{-12}$ for linear system $A$ and $B ; \varepsilon_{=} 10^{-16}$ for linear system $\mathrm{C}$.

\section{Numerical Libraries}

Two numerical libraries were used in this study: PETSc [4] and Hypre [9]. Both provide parallel routines for solving large sparse linear systems. PETSc, the Portable, Extensible Toolkit for Scientific computation, provides a variety of preconditioners and Krylov subspace solvers. This study used Jacobi, Block Jacobi (BJacobi), successive overrelaxation (SOR), and the Additive Schwartz method (ASM) as preconditioners for CG. BJacobi and ASM were implemented with one block per processor. The individual blocks were solved with ILU(0). Hypre [9] in addition provides multigrid preconditioning, and the study also used Hypre's algebraic multigrid method, boomerAMG, as a preconditioner for CG. The study also experimented with the Support Tree Conjugate Gradient (STCG) method [7], as implemented in PETSc [4]. The STCG method is potentially more effective than CG where $\mathbf{A}$ is a generalized Laplacian [7], but relatively little is known about its performance in practical applications.

\section{Test Problems}

The choice of the best iterative solver may strongly depend on the grid size, the aspect ratio of the grid, and physical parameters of the problem. Typically, the convergence rate of the iterative solver is strongly connected to the condition number of the matrix, $\kappa(\mathbf{A})$. Therefore, this study used three sets of data, which try to depict the important role of these characters. 


\subsection{Linear System A}

The FEMWATER code [3] was used to create linear system A. The data set is generated from a pump-and-treat model developed for remediation of contaminated groundwater. The 3-D mesh contains 102,996 nodes, 187,902 elements, and 8 material types, for a total of 31 layers. FEMWATER uses Picard linearization, which produces a symmetric, positive-definite (SPD) linear system. The condition number of the coefficient matrix $\mathbf{A}, \kappa(\mathbf{A})=2.7 \times 10^{6}$.

\subsection{Linear System B}

The WASH123D watershed code was constructed based on its first-principle, physics-based mathematical model [2]. In WASH123D, the cross-section-averaged 1$\mathrm{D}$ diffusive wave equation, the depth-averaged 2-D diffusive wave equation, and the 3-D Richards equation were solved with semi-Lagrangian for canal network flow and overland flow, and Galerkin finite element methods for variably saturated subsurface flow, respectively [2]. Also, the computational domain is discretized with finite element meshes; each element can be assigned with a different material type to account for heterogeneity; and each material may have its own set of physical model parameters. The 3-D subsurface flow in this coupled watershed model is governed by the Richards equation, solved by Picard linearization, but employs a modular software approach.

The 3-D mesh contains 59,409 nodes, 84,996 elements, and includes 17 material types for a total of six layers. Figure 1 shows the 3-D computational mesh of this linear system B.

Similar to the linear system A above, the matrix $\mathbf{B}$ is symmetric and positive definite, with condition number $\kappa(\mathbf{B})=3.9 \times 10^{9}$; thus this linear system is ill-conditioned.

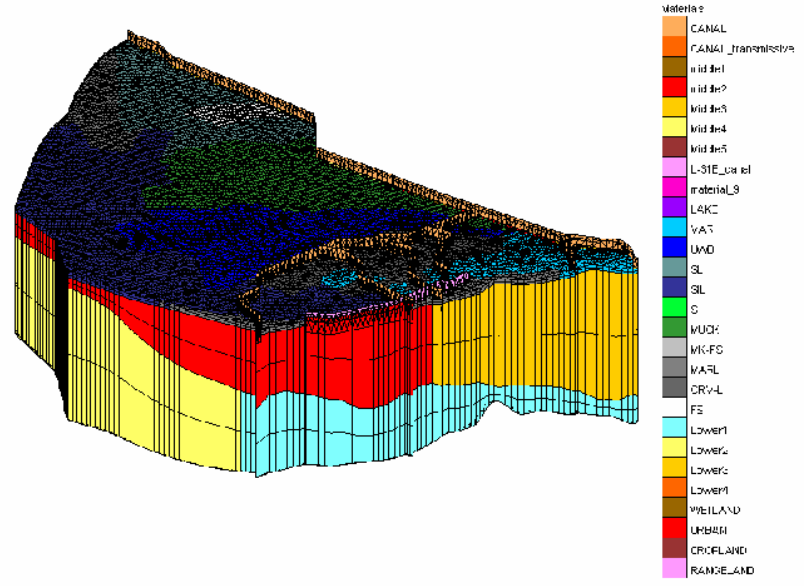

Fig. 1. The 3-D computational mesh of data set B

\subsection{Linear System C}

Linear system $\mathrm{C}$ was also generated by the WASH123 code, from the same model as linear system B, using finer mesh. The 3-D mesh for this system contains 2,124,108 
nodes, 4,018,700 elements, and includes 17 material types for a total of 20 layers. The resulting matrix is symmetric and positive definite.

\section{Test Results}

A C code was written to read these data sets and then call the PETSc functions to create a sparse parallel matrix $\mathbf{A}$ in AIJ format, a parallel vector $\mathbf{b}$, and independent solution xtrue, which was used to compare with solution from PETSc and Hypre for the purpose of verification. The code was compiled and run on a Cray XT4 containing 2,152 compute nodes; each has $2.1 \mathrm{GHz}$ AMD Opteron 64-bit quad-core processors and 8 GBytes of dedicated memory.

Tables 1-3 compare the convergence and performance of different preconditioners for CG and STCG methods, respectively. As expected, preconditioning can significantly increase performance of Krylov subspace solvers. Without preconditioning, the solvers often fail to converge. Both CG and STCG methods using the Jacobi preconditioner are robust solvers for all three linear systems but not necessarily the most efficient. ASM is the most effective preconditioner for linear systems B and C. The ASM preconditioner option -pc_asm_type basic was employed, which uses the full restriction and interpolation operator. The option $-p c \_a s m \_t y p e$ restrict is the PETSc default, but it does not converge for linear systems A, B, or C.

Table 1. Solver time, number of iterations, and residual norm using CG (shaded) and STCG (white) methods for linear systems A (two cores)

\begin{tabular}{|l|l|l|l|l|l|l|}
\hline System & \multicolumn{3}{|c|}{ CG } & \multicolumn{3}{c|}{ STCG } \\
\hline PC & $\begin{array}{l}\text { Residual } \\
\text { Norm } \\
\text { X10 }\end{array}$ & Iteration & $\begin{array}{l}\text { Time } \\
(\mathbf{s e c})\end{array}$ & $\begin{array}{l}\text { Residual } \\
\text { Norm } \\
\mathbf{X 1 0}^{-9}\end{array}$ & $\begin{array}{l}\text { Iteration } \\
\text { Time } \\
(\mathbf{s e c})\end{array}$ \\
\hline None & 1348.94 & 5700 & 39.61 & 1348.94 & 5700 & 41.03 \\
\hline Jacobi & 3.22 & 670 & 4.73 & 3.22 & 670 & 4.90 \\
\hline Bjacobi & 10.20 & 210 & 3.98 & 3.22 & 210 & 4.05 \\
\hline SOR & 4.08 & 272 & 5.34 & 4.08 & 272 & 5.43 \\
\hline ASM & 12.59 & 220 & 5.02 & 12.59 & 220 & 5.08 \\
\hline
\end{tabular}

Table 2. Solver time, number of iterations, and residual norm using CG (shaded) and STCG (white) methods for linear system B (two cores)

\begin{tabular}{|l|l|l|l|l|l|l|}
\hline System & \multicolumn{3}{|c|}{ CG } & \multicolumn{3}{c|}{ STCG } \\
\hline PC & $\begin{array}{l}\text { Residual } \\
\text { Norm } \\
\mathbf{X 1 0}^{-9}\end{array}$ & Iteration & $\begin{array}{l}\text { Time } \\
(\mathbf{s e c})\end{array}$ & $\begin{array}{l}\text { Residual } \\
\text { Norm } \\
\text { X10-9 }\end{array}$ & $\begin{array}{l}\text { Iteration } \\
\text { Time } \\
(\mathbf{s e c})\end{array}$ \\
\hline None & NC & NC & NC & NC & NC & NC \\
\hline Jacobi & 9.05 & 20340 & 76.58 & 9.05 & 20340 & 78.75 \\
\hline Bjacobi & 9.05 & 20340 & 78.11 & 9.05 & 20340 & 80.62 \\
\hline SOR & 6.93 & 18784 & 173.28 & 6.93 & 18784 & 176.00 \\
\hline ASM & 18.93 & 2818 & 34.66 & 18.93 & 2818 & 35.07 \\
\hline
\end{tabular}

Note: NC means not converge. 
Table 3. Solver time, number of iterations, and residual norm using CG (shaded) and STCG (white) methods for linear system C (two cores)

\begin{tabular}{|l|l|l|l|l|l|l|}
\hline System & \multicolumn{3}{|c|}{ CG } & \multicolumn{3}{c|}{ STCG } \\
\hline PC & $\begin{array}{l}\text { Residual } \\
\text { Norm } \\
\mathbf{X 1 0}^{-13}\end{array}$ & Iteration & $\begin{array}{l}\text { Time } \\
(\mathbf{s e c})\end{array}$ & $\begin{array}{l}\text { Residual } \\
\text { Norm } \\
\mathbf{X 1 0}^{-\mathbf{1 3}}\end{array}$ & Iteration & $\begin{array}{l}\text { Time } \\
(\mathbf{s e c})\end{array}$ \\
\hline None & NC & NC & NC & NC & NC & NC \\
\hline Jacobi & 1.37 & 105960 & 17123.40 & 1.37 & 105960 & 17836.93 \\
\hline Bjacobi & 5.50 & 47517 & 18269.13 & 5.50 & 47517 & 18611.86 \\
\hline SOR & 1.21 & 57391 & 22968.26 & 1.21 & 57391 & 23381.96 \\
\hline ASM & 6.24 & 30784 & 15994.27 & 6.24 & 30784 & 16220.26 \\
\hline
\end{tabular}

Note: NC means not converge.

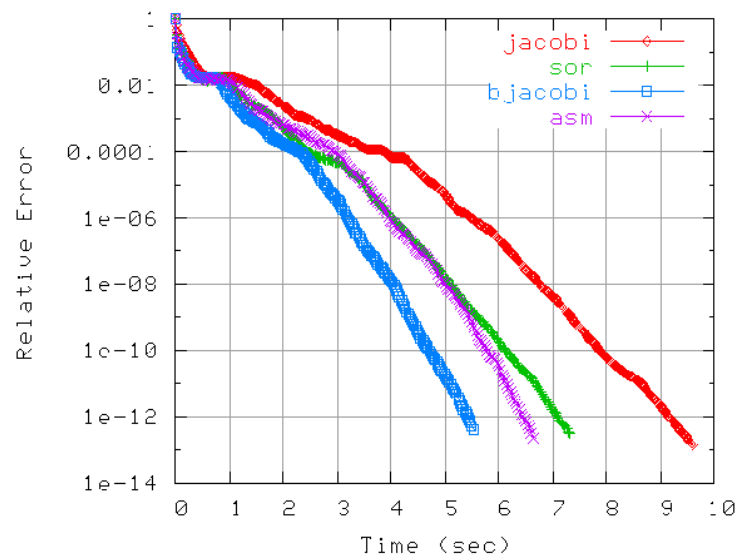

Fig. 2. Relative error versus solver time using CG method for linear system A Relative error Ilb $-\mathbf{A} \mathbf{X}^{\mathbf{k}}\|/\| \mathbf{b}-\mathbf{A} \mathbf{X}^{0} \|$

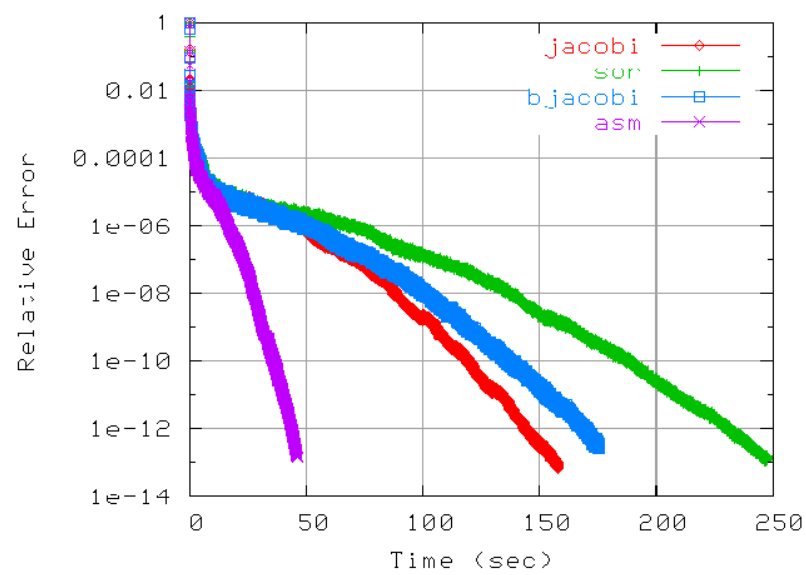

Fig. 3. Relative error versus solver time using CG method for linear system B Relative error Ilb $-\mathbf{A} \mathbf{X}^{\mathrm{k}}\|/\| \mathbf{b}-\mathbf{A} \mathbf{X}^{0} \|$ 


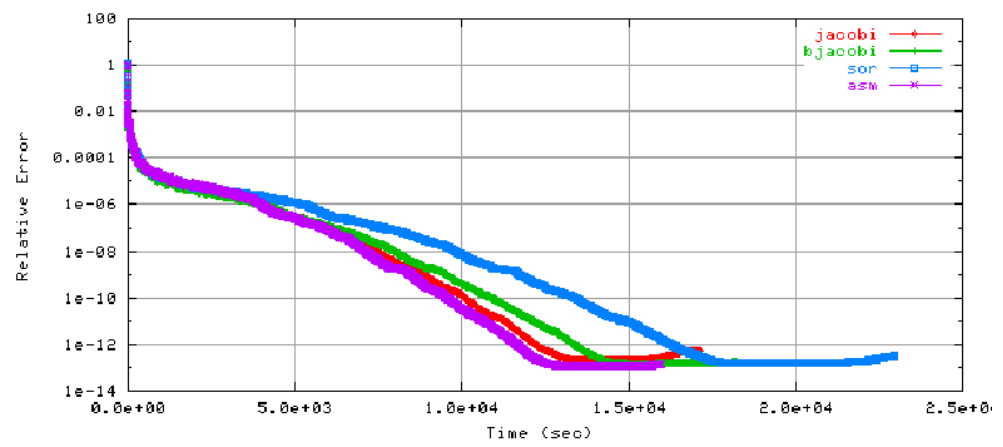

Fig. 4. Relative error versus solver time using CG method for linear system C Relative error IIb $-\mathbf{A} \mathbf{X}^{\mathbf{k}}\|/\| \mathbf{b}-\mathbf{A} \mathbf{X}^{0} \|$

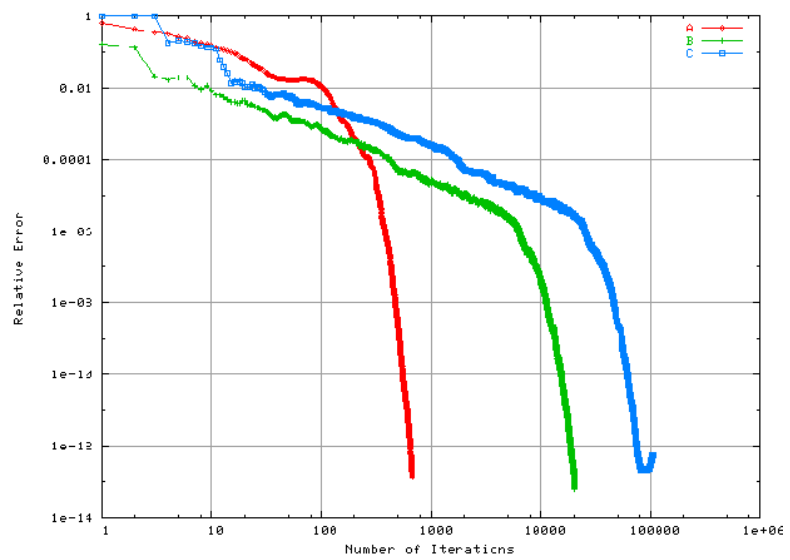

Fig. 5. Relative error versus number of iterations using CG method with Jacobi preconditioner for linear systems A, B, and C. Relative error $\left\|\mathbf{b}-\mathbf{A} \mathbf{X}^{\mathbf{k}}\right\| /\left\|\mathbf{b}-\mathbf{A X}^{\mathbf{0}}\right\|$.

Figures 2-4 compare convergence of the residual norm for different preconditioners using the CG method. For linear system A, Block-Jacobi is the most efficient in terms of solver time, followed by ASM, SOR, and Jacobi. However, for linear systems B, ASM converges in the fewest iterations and requires the least amount of time, followed by the Block-Jacobi, SOR, and Jacobi. For linear system C, ASM converges slightly faster than others, followed by Jacobi, Block-Jacobi, and SOR.

The convergence behavior of the three linear systems is compared in Figure 5 for the Jacobi preconditioner. The behavior of the three systems follows the same pattern; an initial, relatively slow rate of convergence is followed by an abrupt change to a more rapid rate of convergence. The initial stage appears to be approximately linear, or slightly faster, in the number of iterations, while in the latter stage, convergence is faster than quadratic. For these linear systems, which are closely related in terms of the underlying PDE, the initial rates of convergence are very similar, i.e., the error is reduced at the same rate for each linear system. 


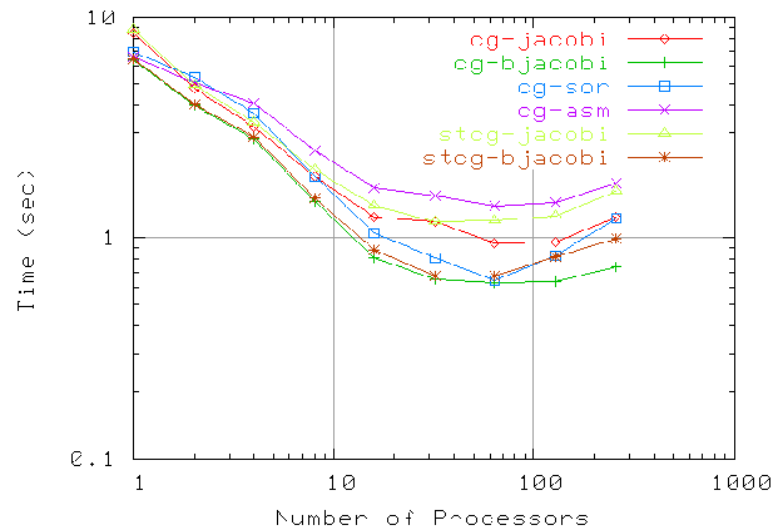

Fig. 6. Solver time using CG and STCG methods combination with Jacobi, Block Jacobi, SOR, and ASM preconditioners for linear system A

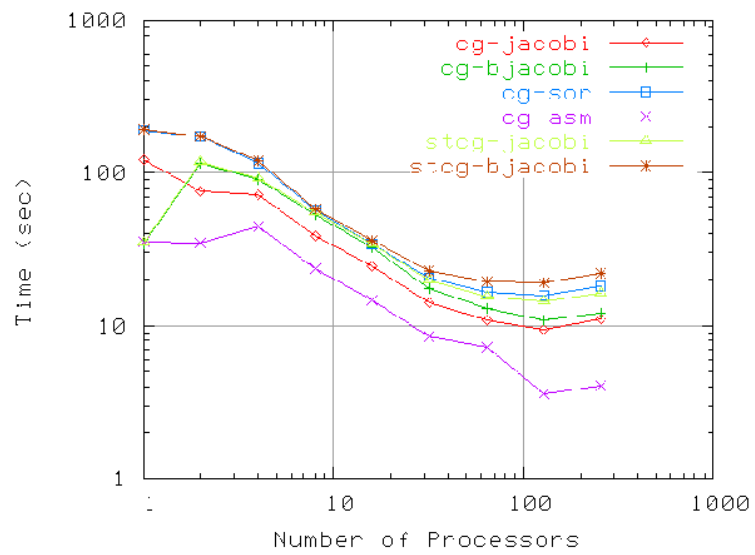

Fig. 7. Solver time using CG and STCG methods combination with Jacobi, Block Jacobi, SOR, and ASM preconditioners for linear system B

Figures 6-8 show the scaling of solver time with the number of processors using CG and STCG methods. For linear system A, the performance scales linearly up to about 16 processors and then flattens out, indicating that fewer than 5000-10,000 nodes per processor results in lower parallel efficiency. The various preconditioners have similar scaling behavior for system A. For linear system B, performance scales erratically in the range from one to four processors but scales linearly in the range from 4 to 32 processors. Beyond 32 processors, the performance flattens out for all preconditioners except ASM, which arguably demonstrates linear scaling from 4 to 128 processors. The number of blocks used in the Block-Jacobi and ASM preconditioners depends on the number of processors; because of this, their solver time running on one processor may be less than that on two, four, or eight processors. 
For linear system C, performance does not scale linearly in the range from one to four processors. This problem is due to on-chip memory contention since four cores share memory in the same node on the Cray XT4 system. The performance scales linearly in the range from 4 to 256 processors.

Figure 9 shows the scaling of solver time with the number of processors using boomerAMG method from Hypre for linear systems A and B. The preliminary results are based on a residual tolerance of $\varepsilon_{=} 10^{-7}$.

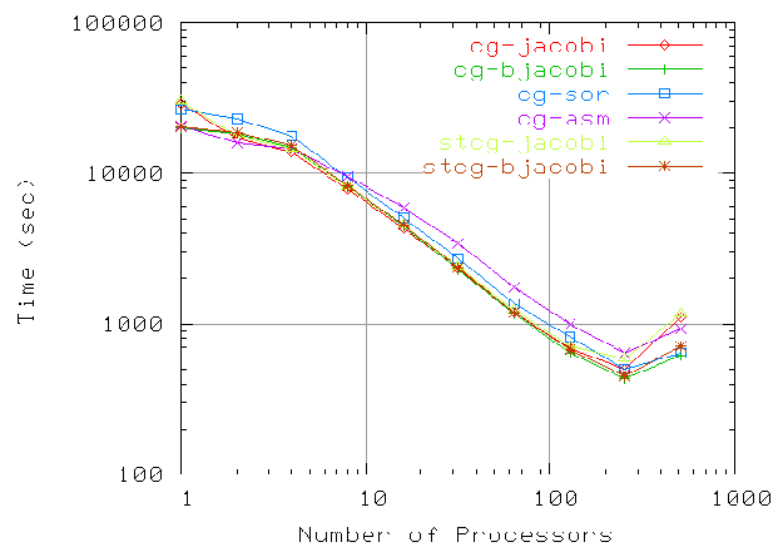

Fig. 8. Solver time using CG and STCG methods combination with Jacobi, Block Jacobi, SOR, and ASM preconditioners for linear system C

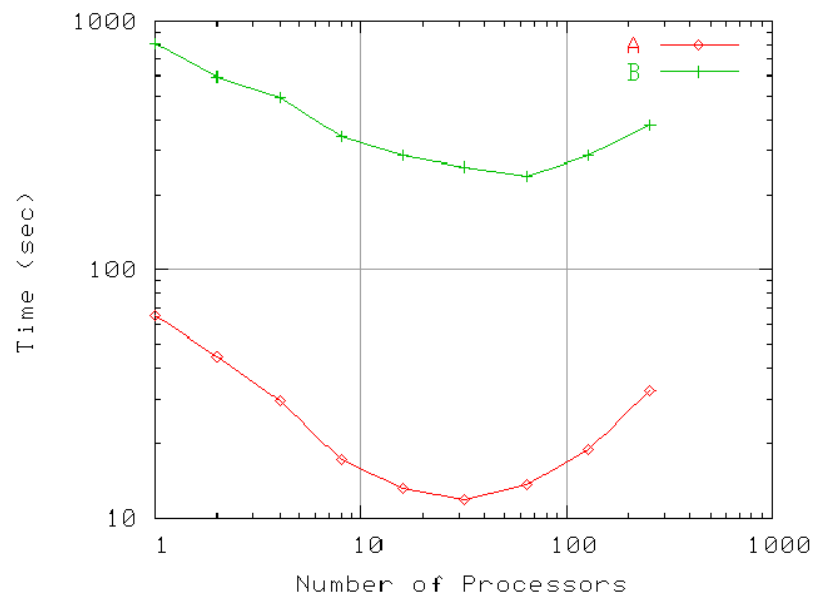

Fig. 9. Solver time using boomerAMG method for linear systems A and B 


\section{Summary and Future Work}

Jacobi-CG appears to be the most robust method for solving moderately illconditioned SPD problems in the range of a few hundred-thousand unknowns. However, it is well known that the number of CG iterations will not scale linearly with matrix dimension. As WASH123 problems become larger, the number of JacobiCG iterations required to achieve a fixed error tolerance will grow, causing the solution time to increase faster than the number of unknowns. This study found that a multilevel preconditioner was effective for linear system B but was not effective with the same preconditioner for the closely related system $\mathrm{C}$. The authors are therefore continuing to experiment with algebraic multigrid and multilevel preconditioners. Even though the cost per iteration of these preconditioners is relatively high for problems in the range of a few hundred-thousand unknowns, they may prove to be more efficient for problems with tens or hundreds of millions of unknowns, where the number of $\mathrm{CG}$ iterations is likely to be prohibitive. The authors also continue to investigate the convergence of CG in time-dependent problems, where a reasonable initial guess is available from the previous time-step, and convergence may be relatively fast.

Acknowledgments. This study was supported by the Department of Defense (DoD) 6.1 Basic research funding in the Civil Works category. It was also supported in part by an allocation of computer time from the DoD High Performance Computing Modernization Program.

\section{References}

1. Cheng, J.R.C., Hunter, R.M., Cheng, H.P., Richards, D.R., Yeh, G.T.: Parallelization of a watershed model|Phase III: Coupled 1-dimensional channel, 2-dimensional overland, and 3dimensional subsurface ows. In: Computational Methods in Water Resources XVI, Copenhagen, Denmark, CMWR CD-ROM, paper 64, June 19-22 (2006)

2. Yeh, G.T., Huang, G., Zhang, F., Cheng, H.P., Lin, H.C., Cheng, J.R., Edris, E.V., Richards, D.R.: An integrated media, integrated processes watershed model WASH123D: Part 1model descriptions and features. In: Computational Methods in Water Resources XVI, Copenhagen, Denmark, CMWR CD-ROM, paper 29, June 19-22 (2006)

3. Lin, H.J., Richards, D.R., Yeh, J., Cheng, J., Cheng, H., Jones, N.L.: FEMWATER: A three-dimensional finite element computer model for simulating density dependent flow and transport, TR CHL-97-12, U.S. Army Engineer Waterways Experiment Station, Vicksburg, MS (1997)

4. Balay, S., Buschelman, K., Gropp, W.D., Kaushik, D., Knepley, M.G., McInnes, L.C., Smith, B.F., Zhang, H.: PETSc (2008), http : / / www. mcs . anl .gov/pet sc

5. Tracy, T.F., Oppe, C.T., Gavali, S.: Testing Parallel Linear Iterative Solvers for Finite Element Groundwater Flow Problems. NAS Technical report; NAS-07-007 (September 2007) 
6. Cheng, C.J.-R., Nguyen, H., Cheng, H.-P(P.), Richards, R.D.: Parallel Performance of the Coupled Watershed System with Multiple_Spatial Domains and Multiple-Temporal Scales. In: PARA2008, 9th International Workshop on State-of-the-Art in Scientific and Parallel Computing, NTNU, Trondheim, Norway, May 13-16 (2008)

7. Gremban, D.K., Miller, L.G., Zagha, M.: PerfoMarco: Performance Evaluation of a New Parallel Preconditioner. In: IPPS 1995: Proceeding of the 9th International Symposium on Parallel Processing (1995)

8. Demmel, J.W.: Applied Numerical Linear Algebra. SIAM, Philadelphia (1997)

9. Falgout, R.D., Meier Yang, U.: hypre: A library of high performance preconditioners. In: Sloot, P.M.A., Tan, C.J.K., Dongarra, J., Hoekstra, A.G. (eds.) ICCS-ComputSci 2002. LNCS, vol. 2331, pp. 632-641. Springer, Heidelberg (2002); also available as LLNL Technical Report UCRL-JC-146175 